\title{
Comparing plasma and faecal measures of steroid hormones in Adelie
}

penguins Pygoscelis adeliae

Ninnes, C.E. ${ }^{1 *}$, Waas, J.R. ${ }^{1}$, Ling, N. ${ }^{1}$, Nakagawa, S. ${ }^{1,2}$, Banks, J.C. ${ }^{1}$, Bell, D. G. ${ }^{1}$, Bright, A. ${ }^{1}$, Carey, P.W. ', Chandler, J. ${ }^{1}$, Hudson, Q.J. Ingram, J.R. ', Lyall, K. ', Morgan, D. ', Stevens, M.I. ${ }^{4}$, Wallace, J. ${ }^{1}$, Möstl, E. ${ }^{5} \quad$ (Authors 5-15 are listed in alphabetical order)

${ }^{1}$ Department of Biological Sciences, University of Waikato, Private Bag 3105, Hamilton, New Zealand

${ }^{2}$ Department of Zoology, University of Otago, PO Box 56, Dunedin, New Zealand

${ }^{3}$ SubAntarctic Foundation for Ecosystems Research, 8 Estuary Road, Christchurch 8061, New Zealand

${ }^{4}$ Allan Wilson Centre for Molecular Ecology and Evolution, Massey University, PB 11-222, Palmerston North, New Zealand; and School of Biological Sciences, Flinders University of South Australia, GPO Box 2100, Adelaide, S.A. 5001, Australia

${ }^{5}$ Institute of Biochemistry, Department of Natural Sciences, University of Veterinary Medicine, A-1210 Vienna, Austria

*Corresponding author:

Current address: Department of Biological Sciences, University of Waikato,

Private Bag 3105, Hamilton, New Zealand

Email: calum.ninnes@gmail.com

Phone: +64 78384466 ext. 8123

Fax: +6478384324 
Abstract. Physiological measurements of both stress and sex hormones are often used to estimate the consequences of natural or human-induced change in ecological studies of various animals. Different methods of hormone measurement exist, potentially explaining variation in results across studies; methods should be cross-validated to ensure they correlate. We directly compared faecal and plasma hormone measurements for the first time in a wild free-living species, the Adelie penguin (Pygoscelis adeliae). Blood and faecal samples were simultaneously collected from individual penguins for comparison, and assayed for testosterone and corticosterone (or their metabolites). Sex differences and variability within each measure, and correlation of values across measures were compared. For both hormones, plasma samples showed greater variation than faecal samples. Males had higher mean corticosterone concentrations than females, but the difference was only statistically significant in faecal samples. Plasma testosterone, but not faecal testosterone, was significantly higher in males than females. Correlation between sample types was poor overall, and weaker in females than in males, perhaps because measures from plasma represent hormones that are both free and bound to globulins, whereas measures from faeces represent only the free portion. Faecal samples also represent a cumulative measure of hormones over time, as opposed to a plasma 'snapshot' concentration. Our data indicate that faecal sampling appears more suitable for assessing baseline hormone concentrations, while plasma sampling may best define immediate responses to environmental events. Consequently, future studies should ensure they select the most appropriate matrix and method of hormone measurement to answer their research questions.

Keywords: Adelie penguin, blood, faecal, corticosterone, testosterone, sex differences. 


\section{Introduction}

Assessing stress in animals, particularly chronic stress (for a discussion of chronic vs. acute stress see Moberg, 2000), is crucial in fields such as animal welfare and conservation biology (Vleck et al., 2000; Möstl and Palme, 2002). Physiological measurements of stress are increasingly being used because legislators and managers require easily quantifiable information on, for example, the effects of human disturbance on wildlife, or species decline (Wikelski and Cooke, 2006). Recently, researchers have found that physiological measurements do not always correlate with other measurements of stress, such as behaviour (Walker et al., 2005a), and that these physiological measurements can reveal additional information that has remained masked in more conventional behavioural studies (Wikelski and Cooke, 2006).

Activity of the sympathetic-adrenal and hypothalamic-pituitary-adrenal systems, which release catecholamines and glucocorticoids respectively, are amongst the most useful physiological parameters to measure in the context of stress assessment. This is because they are triggered (though not exclusively) by stimuli perceived by an animal to be aversive (Broom and Johnson, 1993). Glucocorticoids (e.g. cortisol and corticosterone) function over a longer time period (i.e. they have a slower release and longer half-life) than catecholamines, and may have longer term effects, thus making them more biologically relevant for chronic stress assessment (Broom and Johnson, 1993; von Holst, 1998; Palme et al., 2005). Corticosterone is the main avian glucocorticoid (Ringer, 1976), and has been traditionally measured from blood plasma using radio immunoassays (RIAs) or enzyme immunoassays (EIAs) (Adkins-Regan, 2005). More recently, however, the measurement of corticosterone (and its metabolites), as well as other steroid hormones, from faecal samples has become increasingly popular (Adkins-Regan, 2005); for example, the method has been used 
extensively in recent studies measuring steroid hormones in birds (e.g. Washburn et al., 2003; Sorato and Kotrschal, 2006; Arlettaz et al., 2007; Kralj-Fišer et al., 2007; Cyr and Romero, 2008).

One major benefit of faecal sampling is that it is relatively non-invasive; thus samples can be collected with minimal disturbance to the animal, enabling new possibilities for re-sampling the same individuals without bias from sampling disturbance. Plasma and faecal measures differ, however, in that whilst plasma samples represent a 'snapshot' of circulating hormone concentrations at a specific point in time, faecal measures represent an accumulation of metabolized and excreted hormone over a species-specific time period, representing the intestinal passage time from duodenum to rectum (Palme et al., 1996; von Holst, 1998; Möstl and Palme, 2002). Thus, while plasma measures provide the option to ascertain the maximum concentrations which circulating hormones may reach following a stimulus (which may be of interest in some instances), faecal samples represent an integrated measure of the hormonal response to a stimulus, incorporating duration; it is the integrated response (magnitude and duration) that is biologically important, not magnitude alone (Dallman \& Bhatnager, 2001; Romero, 2004). There are now several papers reviewing how to interpret faecal hormone (or hormone metabolite) measures with respect to plasma measures (e.g. Möstl and Palme, 2002; Millspaugh and Washburn, 2004; Goymann, 2005; Möstl et al., 2005; Palme et al., 2005; Touma and Palme, 2005). A small number of studies have simultaneously measured plasma hormones and faecal hormone metabolites to detect changes following artificial stimulation and suppression in domestic animals (Möstl et al., 1999; Palme et al., 1999; Dehnhard et al., 2003); however, to our knowledge no studies have specifically quantified the relationship between plasma and faecal measures in a natural context, nor examined the consistency of results obtained when plasma hormone and faecal hormone metabolite measures are used 
simultaneously to assess baseline hormone concentrations. Ultimately, plasma and faecal measures appear suited for application to quite different questions. Despite this, there seems to be an assumption that the two measures should produce similar results, as each measure has been used in different studies to examine baseline hormone concentrations in birds (e.g. Groscolas et al., 1986; Arlettaz et al., 2007; Kralj-Fišer et al., 2007; Angelier et al., 2008), with emphasis being placed on the non-invasiveness of faecal sampling as the primary difference between the two methods. However, given the differences between the measures it might be expected that results may differ depending on the method used.

The aim of this study was to investigate, for the first time in a wild free-living species, the variation within and correlations between baseline concentrations of blood plasma measurements and faecal metabolite measurements of two commonly measured steroid hormones: the stress hormone corticosterone, and the sex hormone testosterone, in Adelie penguins (Pygoscelis adeliae). We use 'baseline' as meaning the average hormone concentrations which occur in the animal for the majority of the time at that stage of the life cycle. Thus, 'baseline' may not reflect truly 'basal' concentrations as no animal is free of experiencing a range of stressors during its everyday activities (see Romero, 2002), and chronic stressors can cause 'baseline' hormone levels to be elevated (above basal) long term (e.g. the energetic demand of sustained breeding activities, Angelier et al., 2006). Sex hormones are often quantified in conjunction with corticosterone (e.g. Wingfield et al., 1982; Klukowski et al., 1997; Knapp and Moore, 1997; McQueen et al., 1999; Wada et al., 1999; Sorato and Kotrschal, 2006; Kralj-Fišer et al., 2007; Roberts et al., 2007) to investigate interactions between corticosterone and sex hormones across a breeding season. Differences across the sexes within each measure were also investigated; however, this was done in the context of comparing the consistency of the results generated by the different sample types, 
rather than attempting to elucidate the specific hormonal profiles of each sex. As penguins are commonly used as model species for hormonal and stress studies in birds (e.g. Holberton et al., 1996; McQueen et al., 1998; Fowler, 1999; Walker et al., 2005b; Walker et al., 2006), our results will be particularly valuable for the interpretation of previous studies, and for the undertaking of future studies.

\section{Methods}

\section{Study site}

This study was undertaken at the southern colonies of Cape Bird, Ross Island, Antarctica (ca $77^{\circ} 14$ 'S, $166^{\circ} 28^{\prime} \mathrm{E}$ ) from early November to mid January during the austral summer of 2004/05. The southern colonies are distributed over ice-free rocky substrate at altitudes from 30-70 m above mean sea level and within $500 \mathrm{~m}$ of the McMurdo Sound shoreline. Sixteen sub-colonies were used and marked within the population. The colonies differed in size (ranging from c. 50 pairs to $>1000$ pairs) and in the amount of human disturbance they were exposed to (i.e. ranging from isolated, relatively undisturbed colonies to those that were visited up to twice a day by researchers). These differences ensured that sampled birds varied widely in terms of the amount of stress they experienced. This level of variation was necessary to effectively compare plasma and faecal measures and to ensure that any relationships identified were robust across birds experiencing different social conditions and levels of disturbance.

\section{Sample collection}

Samples were collected at five periods throughout the breeding season (corresponding to courtship, early incubation (males' shift), late incubation (females' shift), guard (post 
hatching), and pre-crèche (just before both parents leave the nest, and chicks form crèches)) to account for temporal variation. At each sampling period, two individuals were sampled from each of the sixteen sub-colonies (where possible, one suspected male and one suspected female, based on observed copulations, occurrence of tread marks on the female's back, and bill size comparisons (Ainley and Emison, 1972; Marchant and Higgins, 1990)). This was done by standing motionless while observing a sub-colony from a distance ( $>5 \mathrm{~m}$ ) until a bird was observed defecating. We compared these data $(n=73)$ to data from a previous year $(n=81)$ in which blood samples were collected within 5 mins by observers initially positioned $>30 \mathrm{~m}$ from the sub-colony; there were no statistically significant differences in the mean plasma corticosterone values $(\mathrm{t}=1.471, \mathrm{p}=0.143)$ or in sample variance $(\mathrm{F}=1.383, \mathrm{p}=0.158)$, so a motionless observer appeared to have little influence on the birds. Following observation of a defecation event, the nest was approached by two observers; one observer removed the bird and carried it a short distance away from the colony for blood sample collection and banding (the bird's head was covered to calm the subject). Simultaneously the other observer would collect the expelled faeces, and cover the eggs/chicks with a wool cap to protect them from predation and the cold. As urinal and faecal excretion are combined in birds, we attempted to collect only the faecal portion from droppings, which was distinguishable by colour. This methodology enabled matched faecal and blood samples to be collected from the same bird, eliminating inter-individual variation in the comparison of results from the two sample types. Samples were collected at various times of the day (0900-2000 h), however circadian rhythms in hormone concentrations were unlikely to have produced any within-day variation due to the continuous $24 \mathrm{~h}$ daylight (Ringer, 1976 and references therein); furthermore, Vleck and Van Hook (2002) found no evidence that circadian rhythms operate in Adelie penguins in this context. 
Faecal samples were collected into cryovials and stored in a cryogenic dry shipper within 2 hours of collection. Blood samples of $0.5-1 \mathrm{~mL}$ were collected by venipuncture from either the brachial vein in the flipper, or from the medial metatarsal vein of the leg using a $3 \mathrm{~mL}$ syringe containing EDTA anticoagulant. This was done by bending the needle about $30^{\circ}$, to allow penetration to occur at a very slight angle. In the flipper, the vein was located by feeling the underside for a groove running laterally down the flipper, then inserting the needle at a very slight angle into the groove. On the inside of the leg, the vein was identified and the needle inserted on a slight angle and only shallowly beneath the skin. All blood samples were collected within 5 min (most well under this time) from first approach of a bird. Studies by both Vleck et al. (2000) and Cockrem et al. (2006) found no detectable plasma corticosterone increase in Adelie penguins within 5 min of approaching a penguin; hence, in this study blood samples represent concentrations not influenced by the sampling disturbance. The blood was kept from freezing until returning to camp $(<2 \mathrm{~h})$, at which point a small blot was made onto an FTA ${ }^{\circledR}$ card (Fitzco/Whatman) (a proprietary medium for DNA storage) for DNA sexing. The remainder of the blood was centrifuged and plasma extracted and transferred into a cryovial. The plasma was stored in a cryogenic dry shipper. Upon returning to New Zealand, all samples were stored at $-80^{\circ} \mathrm{C}$ until analysis. This study was approved by the University of Waikato Animal Ethics Committee and Antarctica New Zealand.

\section{DNA sexing}

A $2 \mathrm{~mm}$ diameter circle of blood sample was punched from the FTA card and transferred into a $1.5 \mathrm{~mL}$ microcentrifuge tube. This was then washed by adding $500 \mu \mathrm{L}$ of TE buffer $(10 \mathrm{mM}$ Tris-HCl, $1 \mathrm{mM}$ EDTA, $\mathrm{pH} 8.0$ ) and vortexing gently. Following this the TE buffer was removed and $20 \mu \mathrm{L}$ of alkaline PEG reagent (see Chomczynski and Rymaszewski, 2006 for reagent details) was added to the sample, which was then shaken at $65^{\circ} \mathrm{C}$ for 5 min to extract 
the DNA. Following extraction, the DNA was amplified by polymerase chain reaction (PCR) following the protocol of Griffiths et al. (1998) using the P2 and P3 primers, which amplify parts of the CHD (chromo-helicase-DNA-binding) gene homologues on both the avian $\mathrm{Z}$ and W sex chromosomes. A Platinum ${ }^{\circledR}$ Taq kit (Invitrogen, Carlsbad, CA, USA) was used for the PCR, and the final reaction conditions were as follows: 1X PCR buffer (minus Mg); $0.2 \mathrm{mM}$ of each dNTP; $1.5 \mathrm{mM} \mathrm{MgCl}_{2} ; 1 \mu \mathrm{M}$ of each primer; 1.0 unit of Taq polymerase; $1 \mu \mathrm{L}$ of the DNA-PEG solution. PCR was performed in a PTC-100 thermal cycler (MJ Research). An initial denaturing step at $95^{\circ} \mathrm{C}$ for 4 min was followed by 30 cycles of $94^{\circ} \mathrm{C}$ for $30 \mathrm{~s}, 48^{\circ} \mathrm{C}$ for $30 \mathrm{~s}$, and $72^{\circ} \mathrm{C}$ for $45 \mathrm{~s}$. On completion of the PCR, 1 unit of HaeIII restriction enzyme (which selectively cuts only the CHD-Z gene) was added to each PCR tube and incubated at $37^{\circ} \mathrm{C}$ for 15 min. The cut DNA was then separated by electrophoresis on a $1 \%$ agarose gel stained with ethidium bromide, producing one band for males (ZZ, 350bp) and two bands for females (ZW, $\sim 350 \mathrm{bp}$ and $\sim 450 \mathrm{bp}$ ). The results of the DNA test were checked against samples in which the sexes were known (from observations of copulations), and matched in all instances.

\section{Extraction of hormones and hormone metabolites from faeces}

Faecal samples were thawed and allowed to reach room temperature. We then weighed 300 mg (where possible) of wet faeces (wet faeces were used as the weight of faecal samples was relatively consistent $3.93 \mathrm{~g} \pm 0.06$ (S.E.)) and transferred it to a test tube, where we added 1.5 $\mathrm{mL}$ (5x sample weight) of double-distilled water, and the sample was vortexed. We added $2.25 \mathrm{~mL}$ (7.5x sample weight) of methanol to the sample (making a $60 \%$ methanol solution), and samples were shaken at $920-980$ orbits per minute (opm) for $30 \mathrm{~min}$. Samples were then centrifuged $\left(3000 \mathrm{~g}, 20^{\circ} \mathrm{C}, 10 \mathrm{~min}\right)$ and $300 \mu \mathrm{L}$ of the supernatant transferred to a $1.5 \mathrm{~mL}$ microcentrifuge tube and diluted with $150 \mu \mathrm{L}$ of assay buffer (trishydroxyaminomethane (Merck 8382) 20 mmol/L; $\mathrm{NaCl}$ (Merck 6404) $0.3 \mathrm{~mol} / \mathrm{L} ; 1 \mathrm{~g} / \mathrm{L}$ bovine serum albumin 
(Sigma A-4503); $1 \mathrm{~mL} / \mathrm{L}$ Tween 80 (Merck 822187)). Extracts were then subjected to enzyme immunoassays (EIAs) to measure the concentrations of immunoreactive tetrahydrocorticosterone and testosterone.

\section{Extraction of hormones from plasma}

Plasma was thawed, brought to room temperature and vortexed. $100 \mu \mathrm{L}$ of plasma was then transferred into a new tube, and $100 \mu \mathrm{L}$ of double-distilled water and $5 \mathrm{~mL}$ of diethyl-ether added. Samples were shaken for $30 \mathrm{~min}$ at the maximum speed possible without the liquid reaching within $1.5 \mathrm{~cm}$ of the top of the test tube. The samples were then frozen, and the liquid diethyl-ether decanted into a new tube, leaving the water and hydrophilic molecules frozen at the bottom. The diethyl-ether was then evaporated under nitrogen, and the samples were re-suspended in $200 \mu \mathrm{L}$ of assay buffer. We then used EIAs on these samples to measure corticosterone and testosterone concentrations.

We initially intended to measure oestrogens in addition to testosterone and corticosterone. We were able to successfully measure metabolites of oestrogens from our faecal samples; however, due to the relatively low concentration of oestrogens in our plasma samples, we did not have sufficient sample volume to conduct an EIA for plasma oestrogens. Thus, because we could not get any data on plasma oestrogens, we could not examine correlations between the sample types for oestrogens, and have not included our data on faecal oestrogens in this paper. 


\section{Enzyme immunoassays}

All assays were group specific, and detected a range of immunoreactive metabolites, as well as the native hormones being examined. Details of cross-reactivity can be found in the reference given for each assay. All samples were assayed in duplicates; the intra-assay variation was calculated from the variation of measurements between duplicates, except when there was a large discrepancy in which case the sample would be assayed again. One sample with low hormone concentrations (low pool) and one sample with high hormone concentrations (high pool) were assayed on every plate, so that inter-assay variation coefficients could be calculated for both the top and the bottom of the standard curve, where variation is greatest. The sensitivity of all assays was below $1 \mathrm{pg} / \mathrm{well}$.

To measure corticosterone metabolites in faeces, an EIA developed by Quillfeldt and Möstl (2003) and validated in Adelie penguins by Nakagawa et al. (2003) was used to measure the principal metabolite tetrahydrocorticosterone (5 $\beta$-pregnane-3 $\alpha, 11 \beta, 21$-triol-20-one). Biotinylated tetrahydrocorticosterone-21-hemisuccinate (HS) was used as the label. The working dilution of the antibody was 1:40,000, and 1:8,000 for the label. The standard curve ranged from 0.82 to $200 \mathrm{pg} /$ well. The intra-assay variation was $7.0 \%$, and the inter-assay variation was $13.8 \%$ and $6.2 \%$ for the low and high pools, respectively.

To measure corticosterone from blood plasma, an EIA for corticosterone-3carboxymethyloxime(CMO):bovine-serum-albumin(BSA) was used, as described by Palme and Möstl (1997). Biotinylated cortisol-3-CMO served as the label. The working dilution of the antibody was $1: 40,000$, and 1:200,000 for the label. The standard curve ranged from 0.82 to $200 \mathrm{pg} / \mathrm{well}$. The intra-assay variation was $6.5 \%$, and the inter-assay variation was $7.1 \%$ and $5.8 \%$ for the low and high pools, respectively. 
For the measurement of testosterone (and metabolites) from both plasma and faeces, an EIA for testosterone-3-CMO:BSA was used, as described by Palme and Möstl (1993).

Biotinylated $5 \alpha$-androstane-3ß,17ß-diol-3-HS served as the label. The working dilution of the antibody was $1: 75,000$, and 1:5,000,000 for the label. The standard curve ranged from 0.33 to $80 \mathrm{pg} /$ well. The intra-assay variation was $8.0 \%$ and the inter-assay variation was $19.6 \%$ and $11.8 \%$ in the low and high pools, respectively.

Apart from the tetrahydrocorticosterone assay which was biologically validated for Adelie penguins (Nakagawa et al., 2003), a lab-based approach was used to validate the assays on the basis of chromatographic mobility of the immunoreactive substances (Cekan, 1979). A faecal sample extract with high hormone concentrations, following a clean up using Sep-pak C18 cartridges, was chromatographed using reverse phase high performance liquid chromatography (HPLC), with a gradient elution and a starting concentration of $50 \%$ methanol, increasing to $75 \%$. Fractions of $0.3 \mathrm{~mL}$ were collected and the immunoreactive substances were analysed by EIA. For testosterone, two main immunoreactive peaks eluted; one at the same position as authentic testosterone, and one eluting much earlier. Therefore, this immunoreactive compound is much more polar and it is likely that the earlier eluting peak is a conjugated form of testosterone. Although this lab-based validation may not be as unequivocal as a biological validation, it provides strong evidence that what was being measured in the testosterone assays was in fact testosterone. Although the tetrahydrocorticosterone assay has been previously validated in Adelie penguins, an HPLC immunogram for it was also undertaken. The dominating immunoreactive peak eluted in the first fractions, with only a small peak eluting at the position of authentic tetrahydrocorticosterone. To verify that this first peak was not caused by interfering 
substances, a second reverse phase HPLC with a gradient elution and a starting concentration of $20 \%$ methanol increasing to $100 \%$ was undertaken. As expected, the two peaks eluted later, showing that the early peaks seen in the first immunogram were not due to lipid interference. This is unnecessary for plasma samples, as the extraction using diethyl-ether removes some cross-reacting steroids, especially those which are conjugated.

\section{Statistical analyses}

Following natural $\log (\ln )$ transformation of all data to achieve normality or near normality, parametric tests were employed. $F$-tests were used to compare the variances between faecal and plasma measures. Within each measure, sex differences were examined using Student's $t$ tests. For each hormone (and hormone metabolites), the relationship between faecal and plasma values was examined across the sexes using linear regression. This correlation was also examined separately at each of the breeding stages and the general trend of the correlation slopes was relatively consistent. However, reduction in sample size and strong sex ratio bias over the periods (e.g. mainly males in early incubation and females in late incubation) greatly reduced statistical power, and hindered meaningful interpretation of the data. Therefore, data were pooled across periods for statistical analyses, and only these results were presented, not the separate analyses. Sex differences should therefore be interpreted with caution, with emphasis on the consistency of the results from each sample type, rather than on the hormonal profiles of each sex. Effect size, rather than statistical significance, was emphasized in interpreting statistical results as recommended by Nakagawa and Cuthill (2007). 


\section{Results}

\section{Plasma corticosterone and faecal tetrahydrocorticosterone}

Variation in plasma corticosterone $(\mathrm{SD}=1.312)$ was greater $(F=4.1, n=121, p<0.001)$ than in faecal tetrahydrocorticosterone $(\mathrm{SD}=0.645)($ Fig. 1). The variation was also greater for plasma corticosterone $(\mathrm{COV}=1.380)$ than for faecal tetrahydrocorticosterone $(\mathrm{COV}=$ 0.582 ) in the untransformed data. No significant difference in plasma corticosterone between sexes was detected $(t=0.664, d f=119, p=0.51)$, although males had a higher mean value than females (Fig. 2a). Males were, however, found to have significantly higher mean faecal tetrahydrocorticosterone than females $(t=3.383, d f=119, p<0.001)$ (Fig. 2b). The relationship between the two measures was examined for each sex (Fig. 3). Males showed a significant $(n=71, p=0.012)$ positive correlation, however this correlation was weak $(r=0.297)$. No significant correlation was found between the two measures in females $(r=-0.166, n=50, p=0.248 ;$ Fig. 3$)$

Untransformed concentrations of plasma corticosterone $(\mathrm{ng} / \mathrm{ml})($ mean $=1.05, \mathrm{SD}=1.44$, range $=0.02-11.36)$ and faecal tetrahydrocorticosterone $(\mathrm{ng} / \mathrm{g})($ mean $=465.04, \mathrm{SD}=270.58$, range $=41.18-1487.70)$ were within the ranges of concentrations which other studies have reported for plasma corticosterone (McQueen et al., 1999; Vleck \& Van Hook, 2002; Vleck et al., 2000) and faecal tetrahydrocorticosterone (Nakagawa et al., 2003) in Adelie penguins.

\section{Plasma testosterone and faecal testosterone}

Plasma testosterone values also showed greater variation $(\mathrm{SD}=1.087)$ than faecal values $(\mathrm{SD}$ $=0.612)(F=3.16, n=121, p<0.001)$ (Fig. 4). Untransformed data displayed the same trend, with plasma testosterone showing greater variation $(\mathrm{COV}=1.963)$ than faecal testosterone $(\mathrm{COV}=0.595)$. The plasma testosterone data showed males to have significantly higher mean 
testosterone than females $(t=2.182, d f=119, p<0.05)$ (Fig. 5a). Males also showed higher mean concentrations of faecal testosterone metabolites than females, however this difference was not significant $(t=0.698, d f=119, p=0.49)$ (Fig. $5 b)$. When the courtship period was excluded (the only period with a balanced sex ratio) the sex difference in plasma testosterone concentrations fell short of reaching statistical significance $(t=1.783, d f=95, p=0.078)$. Positive correlations were detected between plasma testosterone and faecal testosterone for both sexes (Fig. 6). Males displayed a moderately strong correlation $(r=0.531, n=71, p<$ 0.001), whereas the correlation in females was weaker $(r=0.294, n=50, p=0.039)$.

Untransformed concentrations of plasma testosterone $(\mathrm{ng} / \mathrm{ml})($ mean $=0.82, \mathrm{SD}=1.60$, range $=0.04-10.99)$ were comparable to mean total androgen concentrations $(\sim 0.2-4.5 \mathrm{ng} / \mathrm{ml})$ reported by McQueen et al. (1999) for Adelie penguins across several breeding stages. Untransformed concentrations of faecal testosterone (ng/g) were 216.36 (mean) \pm 128.79 (SD) (range $=31.19-843.62)$; to our knowledge no other studies have measured testosterone from faeces in penguins.

\section{Discussion}

Plasma measures for both testosterone and corticosterone displayed greater variation than their faecal equivalents. This is not entirely surprising, given what is already known about these two measures. Circulating plasma concentrations of corticosterone in birds can change rapidly in response to a multitude of physical and psychological stressors (Siegel, 1980; Harvey et al., 1984), and this response can persist for some time afterwards (Harvey et al., 1984). Wingfield et al. (1992) collected serial blood samples over a period of $60 \mathrm{~min}$ from several bird species subjected to 'capture stress', and found in most instances that corticosterone was highest in the final sample, collected at 60 min. Vleck et al. (2000) blood 
sampled Adelie penguins subjected to handling stress over a $30 \mathrm{~min}$ period and found that plasma corticosterone increased by an order of magnitude above baseline concentrations after 30 min. Other studies have produced similar results (e.g. Astheimer et al., 1994; Bears et al., 2003). Therefore, if an individual experienced a stressful event, such as an attempted nest predation or an agonistic encounter (which could easily be missed by an observer due to the scale of the colony and the spatial distribution of our study sub-colonies), shortly before it was chosen for sampling, then the persistence of the corticosterone response to that stressor could confound the sample to be regarded as baseline. In this regard, the circulating corticosterone 'baseline' of an individual should be viewed, rather than a constant line, as a series of peaks and troughs; plasma corticosterone samples only provide a snapshot at an unknown point on that undulating line. Although a major stressor such as capture and restraint may still produce a detectable rise above this 'baseline' concentration, the variable nature of plasma corticosterone concentrations is particularly pertinent when it is the 'baseline' concentrations that are being compared between groups.

Similarly, testosterone secretion can be instigated by social stimuli in a very transitory manner, with circulating concentrations rising in response to an animal's experiences, then declining rapidly after conditions change (as testosterone has a very short half life) (Wingfield, 1994). Thus, 'baseline' plasma testosterone concentrations could also be viewed as highly variable as discussed above for corticosterone plasma concentrations, although perhaps with less chance of influencing baseline plasma samples due to the shorter persistence (shorter half-life) of elevated plasma testosterone concentrations. This point, of the inadequacy of a single plasma sample for making sound inferences about baseline steroid hormone concentrations, has been outlined previously (von Holst, 1998; Harper \& Austad, 2000; Goymann, 2005; Palme et al., 2005), yet studies still report baseline steroid hormone 
concentrations based on single plasma samples (e.g. Vleck et al., 2000; Walker et al., 2005b; Raouf et al., 2006; Ellenberg et al., 2007).

In contrast, because faecal samples represent an accumulation of metabolized and excreted hormone over a species-specific time period (roughly corresponding to intestinal passage time, Palme et al., 1996; the time from an ACTH challenge to the highest concentrations of GC metabolites being excreted was $13 \pm 3 \mathrm{~h}$ in both sexes of Adelie penguins (Nakagawa et al., 2003)), faecal concentrations could be thought of as a 'mean' concentration over that time period, smoothing out the peaks and troughs that might occur in plasma samples. The effects of these differences can be seen in the results acquired from each method. A sex difference was detected for both plasma corticosterone and faecal tetrahydrocorticosterone; however the difference for plasma was not statistically significant whilst the difference for faecal samples was highly significant, most likely due to the differences in variation within each measure. Thus, unless they have very large sample sizes, studies using plasma corticosterone to assess baseline concentrations, of which there are many (see above for some examples), may not appropriately detect general trends that are present, that may have been detected using measures of faecal metabolites. Indeed, Ellenberg et al. (2007) reported that male yellow-eyed penguins (Megadyptes antipodes) had higher baseline plasma corticosterone concentrations than females on average, but the difference was not statistically significant. However, sex differences in baseline concentrations of plasma corticosterone have been detected previously in Magellanic penguins (Spheniscus magellanicus) (Hood et al., 1998), with males having higher levels than females.

Although faecal measures appear to be better suited for measuring baseline hormone concentrations, an aspect of avian excretion must be noted in regards to using faecal samples 
to measure the acute stress response in birds. As urinary glucocorticoid excretion is faster than faecal excretion in birds (Möstl et al., 2005), but urine and faeces are excreted together, two peaks of corticosterone metabolites are observed in excreta: an earlier peak representing urinary excretion, and a later peak representing faecal excretion (Möstl et al., 2005; Rettenbacher et al. 2004). This point is not as important for the measurement of baseline concentrations, but the pattern of excretion must be considered if using faecal samples to measure an acute stress response (see Scheiber et al., 2005). Additionally, the type of food consumed and the digestive physiology of the studied birds must be considered when comparing faecal hormone concentrations between species, or temporally within species in which diet may change (Klasing, 2005). In some bird species (which consume plant materials that are not easily digestible) part of the digesta is diverted into caeca for extended digestion, producing two types of faeces with differing retention times and thus hormonal concentrations (Klasing, 2005). This does not apply to Adelie penguins, in which diet composition is relatively constant over time (during the summer Adelie penguins on Ross Island feed almost exclusively on two micronecktonic species, Euphausia crystallorophias and Pleuragramma antarcticum (Ainley, 2002)), and caecal digestion is absent due to their highly digestible animal prey (Klasing, 2005; Stevens \& Hume, 2004). Faecal mass may also influence hormone measurement from faeces, with faecal hormone concentrations being more diluted in faecal events of larger mass relative to faecal events of smaller mass (Millspaugh \& Washburn, 2004). Drying samples before analysis can help mitigate this problem.

There is also the possibility that retention times could vary with ingestion rate (feeding frequency). Jackson (1992) found that after $18 \mathrm{~h}$ there were no differences in retention times of different sized meals $(350 \mathrm{~g}, 700 \mathrm{~g}, 1000 \mathrm{~g}$ ) fed to king penguins (Aptenodytes patagonicus), and that gut length appeared to be the primary correlate of mean retention times across several 
seabird species (including 3 penguin species) included in her study. Jackson (1992) did find mean retention times in king penguins were longer when fed two 300g meals (fed 10h apart) than when fed one 1000g meal; however this may be expected due to the time lag between meals. Jackson (1992) also reported longer retention times in postmoulting rockhopper penguins (Eudyptes chrysocome) for one food type, but not other food types, compared to prebreeding retention times. This may be a result of the greater energetic demands of moult relative to breeding in penguins (see Jackson, 1992). These results suggest that meal size, or ingestion rate, are unlikely to drastically influence retention times; however this topic is in need of further research across more species. In addition, it should be noted that there are other confounding factors that can potentially influence the results of faecal hormone measurements, such as sample age and sample storage methods (reviewed by Millspaugh \& Washburn, 2004), which were standardised in this study.

There is another difference between plasma and faecal corticosteroid measures that is not always considered, yet which may be highly biologically relevant. In blood, corticosteroids occur in two portions: bound and free. The free portion has been implicated as being the biologically active portion by many researchers, however, there is some evidence for bound hormone having biological activity (reviewed by Breuner \& Orchinik, 2002). Corticosteroidbinding globulin (CBG) binds up to $95 \%$ of circulating glucocorticoids, meaning only a small fraction of circulating glucocorticoids may be biologically active upon target tissues (Sapolsky, 1992). Thus plasma measurements of corticosteroids could potentially have reduced biological relevance without the assessment of bound corticosteroid proportions. In addition, CBG levels appear to be influenced by sex hormones, with oestrogens inducing its synthesis causing higher levels in females (Sapolsky, 1992). Furthermore, Klukowski et al. (1997) suggested that testosterone caused increases of CBG in dark-eyed juncos (Junco 
hyemalis). Hence, sex differences in corticosteroids detected from plasma measurements, in the absence of information on CBG-bound proportions, may have less biological relevance due to the likelihood of sex differences in CBG concentrations. Contrary to this, as only the free portion of circulating glucocorticoids are available for excretion (Breuner \& Orchinik, 2002; Palme et al., 2005), results from faecal measurements reflect only free glucocorticoid concentrations, and thus may be more biologically relevant. This may also be the case for testosterone, which is also bound (with relatively high affinity) by CBG in birds, or bound by sex steroid binding globulin (SSBG) in mammals (Swett \& Breuner, 2008). It must be noted that the role of $\mathrm{CBG}$ is a very active research area, and there is disagreement on the role of CBG in regulating hormone action (reviewed by Breuner \& Orchinik, 2002); however, most researchers in this area agree that hormone action may be regulated by the free portion, and that it is essential to incorporate binding globulins into studies examining hormone action via plasma (Breuner \& Orchinik, 2002; Swett \& Breuner, 2008).

The correlation between plasma and faecal values shown in Figure 3 supports the idea that results may differ depending on which method is used, with plasma corticosterone and faecal tetrahydrocorticosterone correlating significantly (but weakly) in males, while no significant correlation occurred in females. Our results also suggest that the sexes should be examined separately in this species, and care should be taken to identify sex differences in studies of corticosterone in other avian species, a recommendation supported by the identification of sex differences of both basal corticosterone concentrations, and corticosterone acute responses in other bird species (Astheimer et al., 1994; Hood et al., 1998; Bears et al., 2003; Hayward et al., 2006; Wada et al., 2006). 
A significant sex difference was detected in plasma testosterone, but not in faecal testosterone metabolites. This is difficult to interpret given that plasma testosterone showed more variation than its faecal equivalent. Potentially, due to its short half life, transient elevations in circulating testosterone could produce very high concentrations in plasma samples, whilst having only a small influence on the faecal concentration (due to the short duration of elevated concentrations). This could cause a significant difference to be detected from plasma that is not actually present when examined over a longer time period, which the faecal samples represent. Alternatively, as samples were collected at multiple times over the breeding season, there may be a period of elevated testosterone in males where plasma concentrations in some birds reached very high concentrations, having a strong influence on the mean plasma testosterone concentrations for males. Indeed, Groscolas et al. (1986) recorded elevations of plasma testosterone several fold above baseline in male Adelie penguins during the courtship/copulation period. In our study, sex differences could not be examined separately at each period due to lack of power and strong bias in sex ratios within periods (e.g. males and females alternate incubation duties; the male undertakes the early incubation duties for approximately 2 weeks, then the female takes the following 2-3 weeks during the late incubation period (Ainley, 2002)). However, when the courtship period was excluded (the only period with a balanced sex ratio) there was no significant sex difference in plasma testosterone concentrations, supporting the results reported by Groscolas et al. (1986) that male testosterone concentrations are greatly elevated during courtship. Our data suggest that, following courtship, male testosterone concentrations subsequently decline to levels which are not significantly different from female testosterone concentrations.

It is also possible that the lack of a statistically significant sex difference in faecal testosterone metabolites is caused by the co-measurement of the metabolites of dehyrdoepiandrosterone 
(DHEA, a weak but abundant androgen produced by the adrenal cortex in both sexes) by the testosterone assay. This was found to be probable in some primate species due to the high concentration of circulating DHEA and the similar nature of their testosterone and DHEA metabolites (Möhle et al., 2002); however, co-measurement of the metabolites of testosterone and DHEA is unlikely to pose a problem in other primate groups (e.g. Callitrichids) due to their much lower levels of DHEA (Möhle et al., 2002). To our knowledge the issue of comeasuring metabolites of testicular origin and of DHEA using faecal androgen assays has yet to be investigated in birds, and is an area in need of further study.

The correlation between plasma testosterone and faecal testosterone metabolites was stronger than that between plasma corticosterone and faecal tetrahydrocorticosterone, with males showing a moderate and females a weak correlation. Interestingly, females showed a significant correlation between the two testosterone measures, but the effect size was much smaller than that of the male correlation. As, for both hormones, the correlation appears to breakdown in females compared to that seen in males, this reinforces that the sexes should be interpreted separately, and that different results would be reported depending on which method is used.

This study directly compared, for the first time in a wild species, the results produced by two commonly used sampling methods for two frequently measured hormones, using individually matched samples. As faecal samples displayed less variation than plasma samples for the hormones examined, and given the potential fluctuations in circulating concentrations that these hormones can exhibit, faecal sampling appears to be a more suitable method for the assessment of baseline concentrations in this species and possibly other bird species given the highly conserved nature of the HPA stress response. With the additional virtues of faecal 
sample collection being non-invasive, and faecal samples representing only the biologically active portion of circulating corticosteroids, this sampling method could facilitate more accurate studies of baseline hormone data and of chronic stress. However, plasma sampling may still be useful for mapping the acute stress response. Thus researchers should recognize the distinction between plasma and faecal measures, and select the method that is most appropriate to their question (e.g. using faecal measures for studies comparing baseline hormone concentrations or examining chronic stress, and serial plasma samples for mapping the curve of the acute stress response). Care should also be taken to examine differences between the sexes, as there appears to be pronounced differences in circulating concentrations, and correlations between the two measures. These results contribute to the current body of literature advocating for more care and consideration in choice of sampling method, and inspection of sex differences.

\section{Acknowledgements}

We thank those individuals within Antarctica New Zealand who supported our event, especially staff at Scott Base and field support staff. Thanks to the staff at the Institute for Biochemistry, University of Veterenary Medicine, Vienna, who helped conduct the EIAs. We thank Dick Wilkins and Raewyn Towers for help with the molecular sexing of samples, and Karen Nutt for providing the necessary primers. Thanks to Rupert Plame for helpful comments on earlier drafts of this manuscript, and to Bruce Patty for technical support. We thank Education New Zealand, The Perry Foundation, and the University of Waikato for financial assistance. We thank anonymous reviewers for helpful comments on this manuscript. 


\section{References}

Adkins-Regan, E., 2005. Hormones and animal social behavior. Princeton University Press, Princeton.

Ainley, D. G., 2002. The Adelie penguin: bellwether of climate change. Columbia University Press, New York.

Ainley, D. G., Emison, W., 1972. Sexual size dimorphism in Adelie penguins. Ibis 114, 267271.

Angelier, F., Bost, C. A., Giraudeau, M., Bouteloup, G., Dano, S. Chastel, O., 2008. Corticosterone and foraging behavior in a diving seabird: The Adelie penguin, Pygoscelis adeliae. Gen. Comp. Endocrinol. 156, 134-144.

Angelier, F., Shaffer, S. A., Weimerskirch, H. Chastel, O., 2006. Effect of age, breeding experience and senescence on corticosterone and prolactin levels in a long-lived seabird: The wandering albatross. Gen. Comp. Endocrinol. 149, 1-9.

Arlettaz, R., Patthey, P., Baltic, M., Leu, T., Schaub, M., Palme, R. Jenni-Eiermann, S., 2007. Spreading free-riding snow sports represent a novel serious threat for wildlife. Proc. R. Soc. Biol. Sci. B 274, 1219-1224.

Astheimer, L. B., Buttemer, W. A. Wingfield, J. C., 1994. Gender and seasonal differences in the adrenocortical-response to ACTH challenge in an arctic passerine, Zonotrichia leucophrys gambelii. Gen. Comp. Endocrinol. 94, 33-43.

Bears, H., Smith, J. N. M. Wingfield, J. C., 2003. Adrenocortical sensitivity to stress in darkeyed juncos (Junco hyemalis oregonus) breeding in low and high elevation habitat. Ecoscience 10, 127-133.

Breuner, C. W. Orchinik, M., 2002. Plasma binding proteins as mediators of corticosteroid action in vertebrates. J. Endocrinol. 175, 99-112.

Broom, D. M., Johnson, K. G., 1993. Stress and animal welfare. Chapman \& Hall, London. 
Cekan, S. Z., 1979. On the assessment of validity of steroid radioimmunoassays. J. Steroid Biochem. 11, 1629-1634.

Chomczynski, P., Rymaszewski, M., 2006. Alkaline polyethylene glycol-based method for direct PCR from bacteria, eukaryotic tissue samples, and whole blood. Biotechniques 40, 454-458.

Cockrem, J. F., Potter, M. A. Candy, E. J., 2006. Corticosterone in relation to body mass in Adelie penguins (Pygoscelis adeliae) affected by unusual sea ice conditions at Ross Island, Antarctica. Gen. Comp. Endocrinol. 149, 244-252.

Cyr, N. E. Romero, L. M., 2008. Fecal glucocorticoid metabolites of experimentally stressed captive and free-living starlings: Implications for conservation research. Gen. Comp. Endocrinol. 158, 20-28.

Dallman, M. F. Bhatnager, S., 2001. Chronic stress and energy balance: role of the hypothalamic-pituitary-adrenal axis. In: McEwen B. S. Goodman H. M. (ed.), Handbook of physiology; section 7: The endocrine system; volume IV: Coping with the environment: neural and endocrine mechanisms. Oxford University Press, pp. 197210.

Dehnhard, M., Schreer, A., Krone, O., Jewgenow, K., Krause, M., Grossmann, R., 2003. Measurement of plasma corticosterone and faecal glucocorticoid metabolites in the chicken (Gallus domesticus), the great cormorant (Phalacrocorax carbo), and the goshawk (Accipiter gentilis). Gen. Copm. Endocrinol. 131, 345-352.

Ellenberg, U., Setiawan, A. N., Cree, A., Houston, D. M. Seddon, P. J., 2007. Elevated hormonal stress response and reduced reproductive output in yellow-eyed penguins exposed to unregulated tourism. Gen. Comp. Endocrinol. 152, 54-63.

Fowler, G. S., 1999. Behavioral and hormonal responses of Magellanic penguins (Spheniscus magellanicus) to tourism and nest site visitation. Biol. Conservat. 90, 143-149. 
Goymann, W., 2005. Noninvasive monitoring of hormones in bird droppings - Physiological validation, sampling, extraction, sex differences, and the influence of diet on hormone metabolite levels. Ann. New York Acad. Sci. 1046, 35-53.

Griffiths, R., Double, M. C., Orr, K. Dawson, R. J. G., 1998. A DNA test to sex most birds. Mol. Ecol. 7, 1071-1075.

Groscolas, R., Jallageas, M., Goldsmith, A. Assenmacher, I., 1986. The endocrine control of reproduction and molt in male and female emperor (Aptenodytes forsteri) and Adelie (Pygoscelis adeliae) penguins. Gen. Comp. Endocrinol. 62, 43-53.

Harper, J. M. Austad, S. N., 2000. Fecal glucocorticoids: a noninvasive method of measuring adrenal activity in wild and captive rodents. Physiol. Biochem. Zool. 73, 12-22.

Harvey, S., Phillips, J. G., Rees, A. Hall, T. R., 1984. Stress and adrenal function. J. Exp. Zool. 232, 633-645.

Hayward, L. S., Richardson, J. B., Grogan, M. N. Wingfield, J. C., 2006. Sex differences in the organizational effects of corticosterone in the egg yolk of quail. Gen. Comp. Endocrinol. 146, 144-148.

Holberton, R. L., Helmuth, B. Wingfield, J. C., 1996. The corticosterone stress response in gentoo and king penguins during the non-fasting period. Condor 98, 850-854.

Hood, L. C., Boersma, P. D. Wingfield, J. C., 1998. The adrenocortical response to stress in incubating Magellanic penguins (Spheniscus magellanicus). Auk 115, 76-84.

Jackson, S., 1992. Do seabird gut sizes and mean retention times reflect adaptation to diet and foraging method? Physiol. Zoo. 65, 674-697.

Klasing, K. C., 2005. Potential impact of nutritional strategy on noninvasive measurements of hormones in birds. Ann. New York Acad. Sci.1046, 5-16.

Klukowski, L. A., Cawthorn, J. M., Ketterson, E. D. Nolan, V., 1997. Effects of experimentally elevated testosterone on plasma corticosterone and corticosteroid- 
binding globulin in dark-eyed juncos (Junco hyemalis). Gen. Comp. Endocrinol. 108, $141-151$.

Knapp, R., Moore, M. C., 1997. Male morphs in tree lizards have different testosterone responses to elevated levels of corticosterone. Gen. Comp. Endocrinol. 107, 273-279.

Kralj-Fišer, S., Scheiber, I. B. R., Blejec, A., Moestl, E. Kotrschal, K., 2007. Individualities in a flock of free-roaming greylag geese: behavioral and physiological consistency over time and across situations. Horm. Behav.51, 239-248.

Marchant, S., Higgins, P. J., 1990. Handbook of Australian, New Zealand \& Antarctic birds. Oxford University Press, Melbourne.

McQueen, S. M., Davis, L. S. Young, G., 1998. The reproductive endocrinology of Fiordland crested penguins Eudyptes pachyrhynchus. Emu 98, 127-131.

McQueen, S. M., Davis, L. S. Young, G., 1999. Sex steroid and corticosterone levels of Adelie penguins (Pygoscelis adeliae) during courtship and incubation. Gen. Comp. Endocrinol. 114, 11-18.

Millspaugh, J. J., Washburn, B. E., 2004. Use of fecal glucocorticold metabolite measures in conservation biology research: considerations for application and interpretation. Gen. Comp. Endocrinol. 138, 189-199.

Moberg, G. P., 2000. Biological response to stress: implications for animal welfare. In: Moberg G. P. Mench J. A. (ed.), The biology of animal stress: basic principles and implications for animal welfare. CABI Publishing, Wallingford, pp. 1-21.

Möhle, U., Heistermann, M., Palme, R. Hodges, J. K., 2002. Characterization of urinary and fecal metabolites of testosterone and their measurement for assessing gonadal endocrine function in male nonhuman primates. Gen. Comp. Endocrinol. 129, 135145. 
Möstl, E., Messmann, S., Bagu, E., Robia, C., Palme, R., 1999. Measurement of glucocorticoid metabolite concentrations in faeces of domestic livestock. J. Vet. Med. A 46, 621-631.

Möstl, E., Palme, R., 2002. Hormones as indicators of stress. Domest. Anim. Endocrinol. 23, $67-74$.

Möstl, E., Rettenbacher, S. Palme, R., 2005. Measurement of corticosterone metabolites in birds' droppings: an analytical approach. Ann. New York Acad. Sci. 1046, 17-34.

Nakagawa, S., Cuthill, I. C., 2007. Effect size, confidence interval and statistical significance: a practical guide for biologists. Biol. Rev. 82, 1-15.

Nakagawa, S., Möstl, E. Waas, J. R., 2003. Validation of an enzyme immunoassay to measure faecal glucocorticoid metabolites from Adelie penguins (Pygoscelis adeliae): a noninvasive tool for estimating stress? Polar Biol. 26, 491-493.

Palme, R., Fischer, P., Schildorfer, H. Ismail, M. N., 1996. Excretion of infused C-14-steroid hormones via faeces and urine in domestic livestock. Anim. Reprod. Sci. 43, 43-63.

Palme, R., Möstl, E., 1993. Biotin-streptavidin enzyme immunoassay for the determination of oestrogens and androgens in boar faeces. Proceedings of the 5th Symposium on the Analysis of Steroids 111-117.

Palme, R., Möstl, E., 1997. Measurement of cortisol metabolites in faeces of sheep as a parameter of cortisol concentration in blood. Zeitschrift Fur SaugetierkundeInternational Journal of Mammalian Biology 62 suppl. 2, 192-197.

Palme, R., Rettenbacher, S., Touma, C., El-Bahr, S. M. Möstl, E., 2005. Stress hormones in mammals and birds - comparative aspects regarding metabolism, excretion, and noninvasive measurement in fecal samples. Ann. New York Acad. Sci. 1040, 162-171. 
Palme, R., Robia, Ch., Messmann, S., Hofer, J., Möstl, E., 1999. Measurement of faecal cortisol metabolites in ruminants: a non-invasive parameter of adrenocortical function. Wien. Tierärztl. Mschr. 86, 237-241.

Quillfeldt, P., Möstl, E., 2003. Resource allocation in Wilson's storm-petrels Oceanites oceanicus determined by measurement of glucocorticoid excretion. Acta ethologica 5, 115-122.

Raouf, S. A., Smith, L. C., Brown, M. B., Wingfield, J. C. Brown, C. R., 2006.

Glucocorticoid hormone levels increase with group size and parasite load in cliff swallows. Anim. Behav. 71, 39-48.

Rettenbacher, S., Möstl, E., Hackl, R., Ghareeb, K., Palme, R., 2004. Measurement of corticosterone metabolites in chicken droppings. Br. Poult. Sci. 45, 704-711.

Ringer, R. K., 1976. Adrenals. In: Sturkie P. D. (ed.), Avian physiology. Springer-Verlag, New York, pp. 372-382.

Roberts, M. L., Buchanan, K. L., Hasselquist, D. Evans, M. R., 2007. Effects of testosterone and corticosterone on immunocompetence in the zebra finch. Horm. Behav. 51, 126134.

Romero, L. M., 2002. Seasonal changes in plasma glucocorticoid concentrations in free-living vertebrates. Gen. Comp. Endocrinol. 128, 1-24.

Romero, L. M., 2004. Physiological stress in ecology: lessons from biomedical research. Trends Ecol. Evol. 19, 249-255.

Sapolsky, R. M., 1992. Neuroendocrinology of the stress-response. In: Becker J. B., Breedlove S. M. Crews D. (ed.), Behavioral endocrinology. MIT Press, Cambridge, pp. 287-324. 
Scheiber, I. B. R., Kralj, S. Kotrschal, K., 2005. Sampling effort/frequency necessary to infer individual acute stress responses from fecal analysis in greylag geese (Anser anser). Ann. New York Acad. Sci. 1046, 154-167.

Siegel, H. S., 1980. Physiological stress in birds. Bioscience 30, 529-534.

Sorato, E., Kotrschal, K., 2006. Hormonal and behavioural symmetries between the sexes in the northern bald ibis. Gen. Comp. Endocrinol. 146, 265-274.

Stevens, C. E. Hume, I. D., 2004. Comparative physiology of the vertebrate digestive system ed. Cambridge University Press, Cambridge.

Swett, M. B. Breuner, C. W., 2008. Interaction of testosterone, corticosterone and corticosterone binding globulin in the white-throated sparrow (Zonotrichia albicollis). Comp. Biochem. Physiol. a-Molecular \& Integrative Physiology 151, 226-231.

Touma, C., Palme, R., 2005. Measuring fecal glucocorticoid metabolites in mammals and birds: the importance of validation. Ann. New York Acad. Sci.1046, 54-74.

Vleck, C. M., Van Hook, J. A., 2002. Absence of daily rhythms of prolactin and corticosterone in Adelie penguins under continuous daylight. Condor 104, 667-671.

Vleck, C. M., Vertalino, N., Vleck, D. Bucher, T. L., 2000. Stress, corticosterone, and heterophil to lymphocyte ratios in free-living Adelie penguins. Condor 102, 392-400. von Holst, D., 1998. The concept of stress and its relevance for animal behavior. Adv. Stud. Behav. 27, 1-131.

Wada, H., Moore, I. T., Breuner, C. W. Wingfield, J. C., 2006. Stress responses in tropical sparrows: comparing tropical and temperate Zonotrichia. Physiol. Biochem. Zool. 79, 784-792.

Wada, M., Shimizu, T., Kobayashi, S., Yatani, A., Sandaiji, Y., Ishikawa, T. Takemure, E., 1999. Behavioral and hormonal basis of polygynous breeding in male bush warblers (Cettia diphone). Gen. Comp. Endocrinol. 116, 422-432. 
Walker, B. G., Boersma, P. D. Wingfield, J. C., 2005a. Physiological and behavioral differences in Magellanic penguin chicks in undisturbed and tourist-visited locations of a colony. Conservat. Biol. 19, 1571-1577.

Walker, B. G., Boersma, P. D. Wingfield, J. C., 2006. Habituation of adult Magellanic penguins to human visitation as expressed through behavior and corticosterone secretion. Conservat. Biol. 20, 146-154.

Walker, B. G., Wingfield, J. C. Boersma, P. D., 2005b. Age and food deprivation affects expression of the glucocorticosteroid stress response in Magellanic penguin (Spheniscus magellanicus) chicks. Physiol. Biochem. Zool. 78, 78-89.

Washburn, B. E., Millspaugh, J. J., Schulz, J. H., Jones, S. B. Mong, T., 2003. Using fecal glucocorticoids for stress assessment in mourning doves. Condor 105, 696-706.

Wikelski, M., Cooke, S. J., 2006. Conservation physiology. Trends Ecol. Evol. 21, 38-46.

Wingfield, J. C., 1994. Hormone-behavior interactions and mating systems in male and female birds. In: Short R. V. Balaban E. (ed.), The differences between the sexes. Cambridge University Press, New York, pp. 303-330.

Wingfield, J. C., Smith, J. P. Farner, D. S., 1982. Endocrine responses of white-crowned sparrows to environmental stress. Condor 84, 399-409.

Wingfield, J. C., Vleck, C. M. Moore, M. C., 1992. Seasonal changes of the adrenocortical response to stress in birds of the Sonoran Desert. J. Exp. Zool. 264, 419-428. 


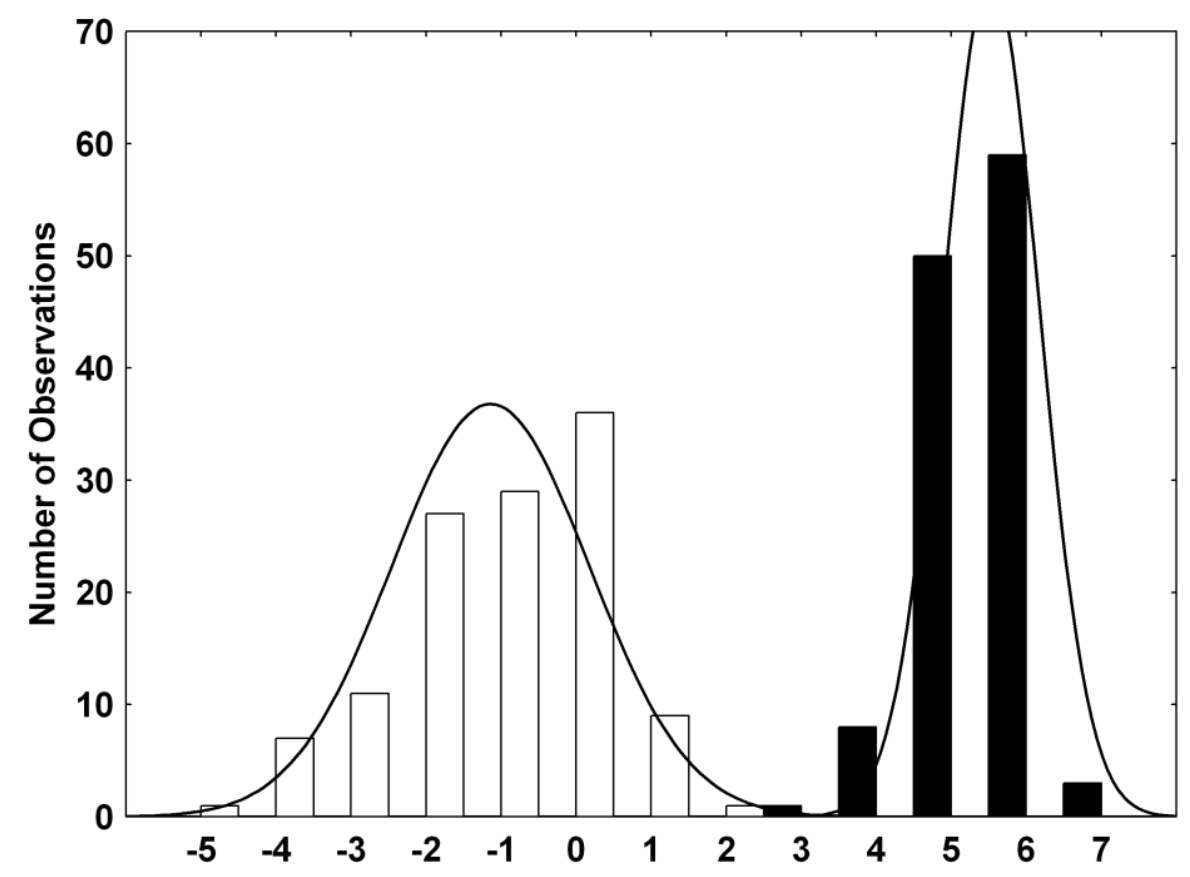

Fig 1: Distributions of $\ln$ plasma corticosterone (ng/mL, open bars) and ln faecal tetrahydrocorticosterone (ng/g, solid bars)
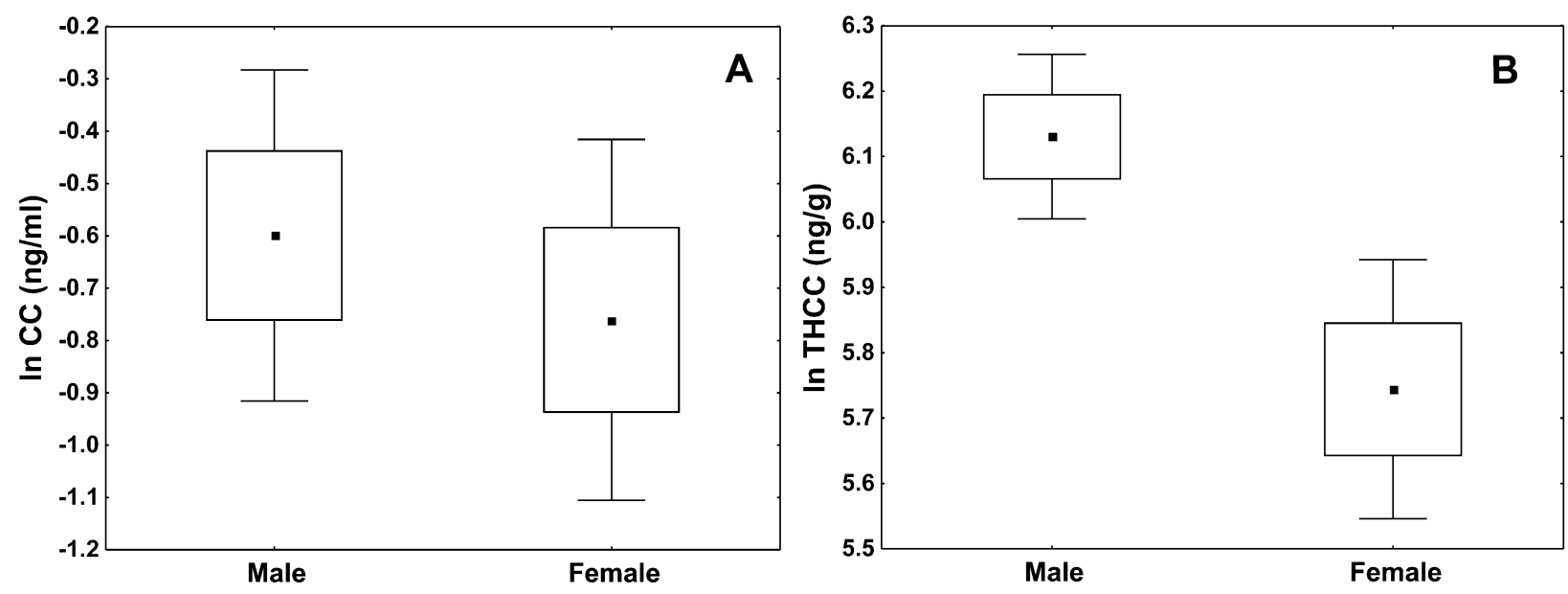

Fig 2: Male and female (A) ln plasma corticosterone levels and (B) ln faecal tetrahydrocorticosterone levels. Values are means $\pm 1 \mathrm{SE}$ (boxes) and 95\% confidence intervals (whiskers) 


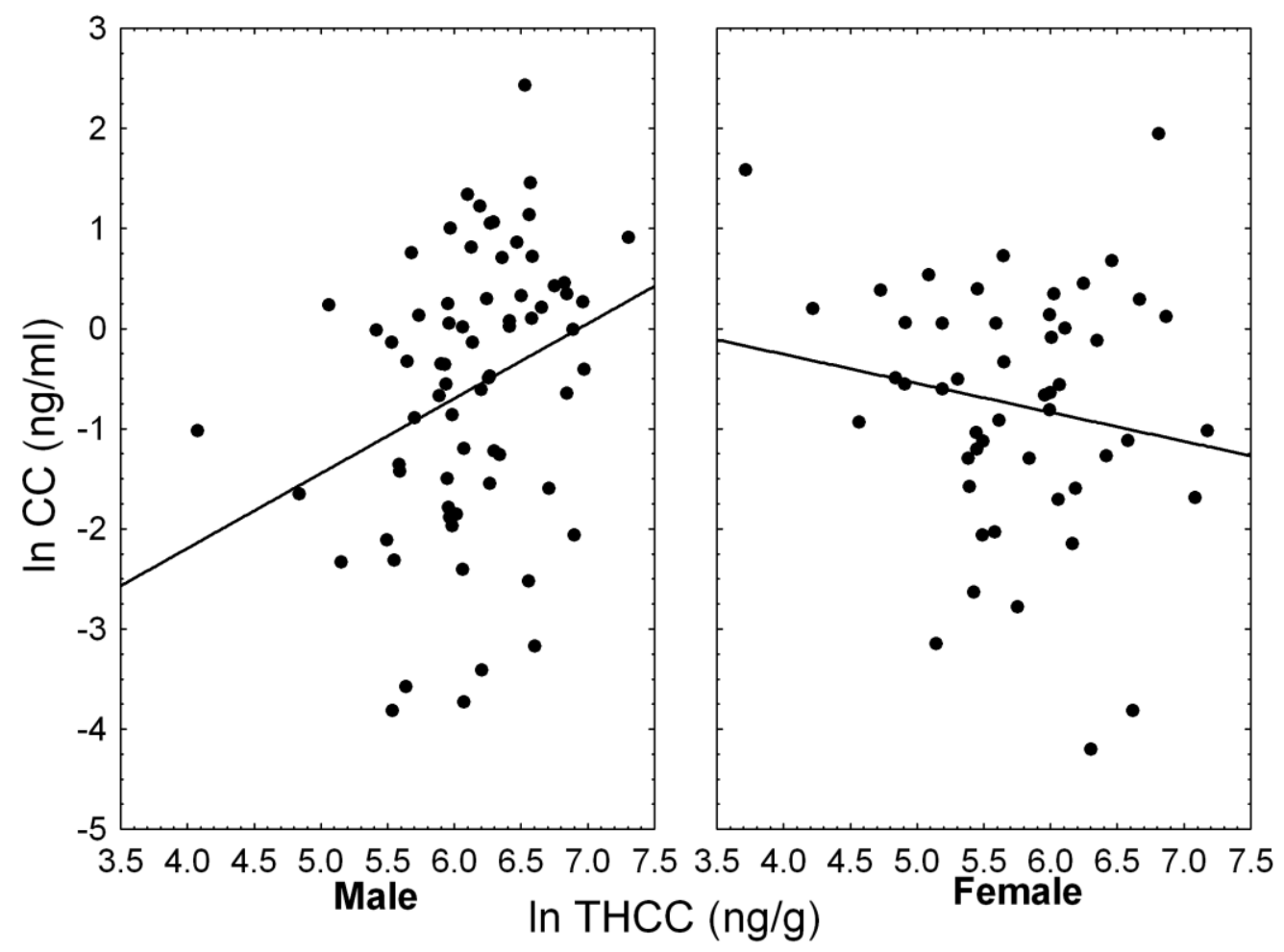

Fig 3: Correlation of $\ln$ plasma corticosterone (CC) and ln faecal tetrahydrocorticosterone (THCC) across the sexes. Male: $y=-5.187+0.748^{*} x$; Female: $y=0.904-0.290 * x$

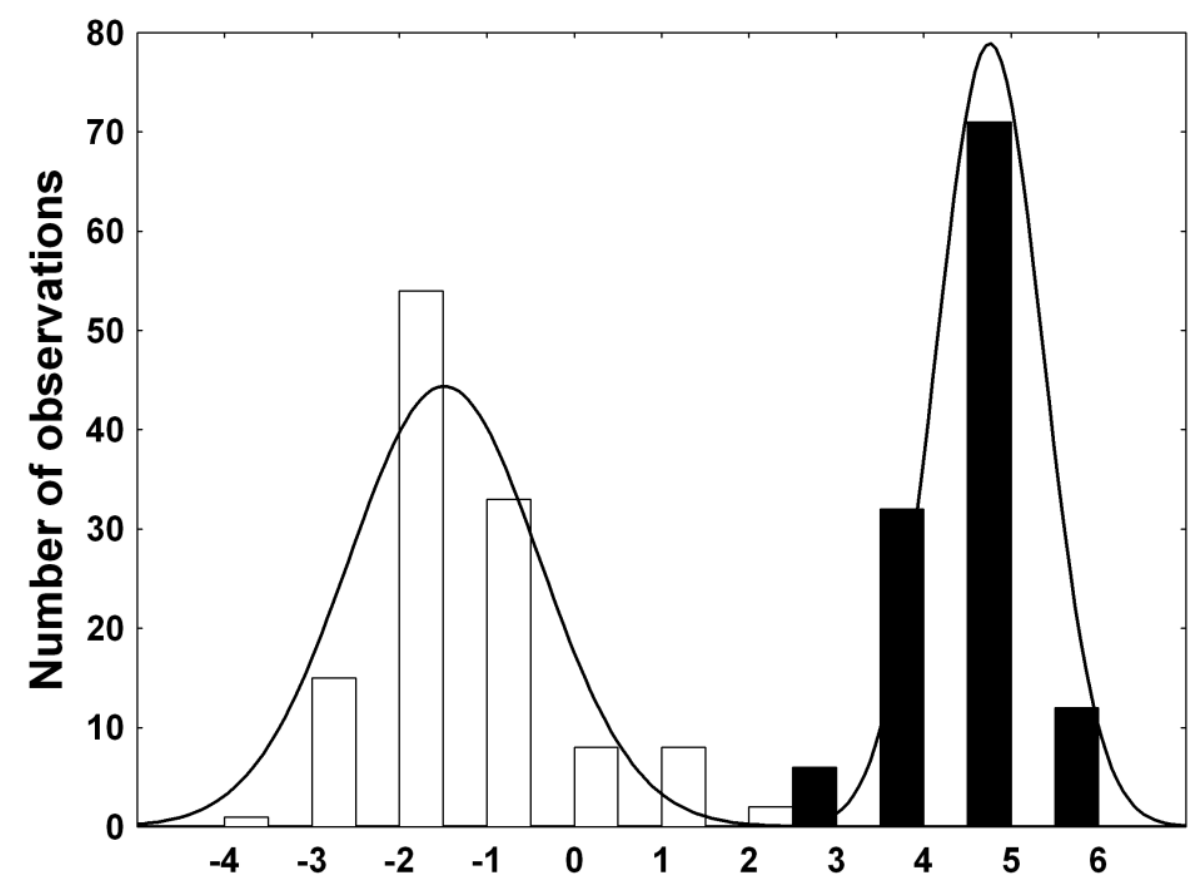

Fig 4: Distributions of ln plasma testosterone (ng/mL, open bars) and ln faecal testosterone (ng/g, solid bars) 

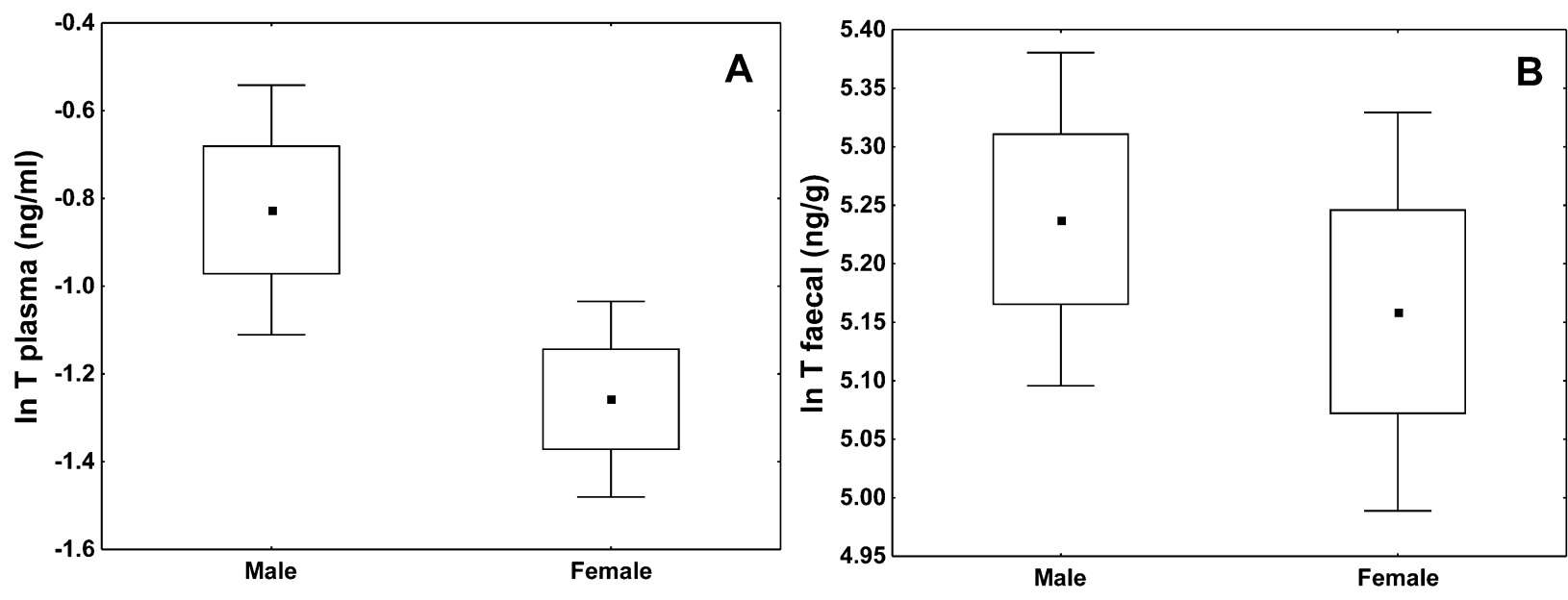

Fig 5: Male and female (A) ln plasma testosterone levels and (B) ln faecal testosterone levels. Values are means $\pm 1 \mathrm{SE}$ (box) and 95\% confidence intervals (whiskers)

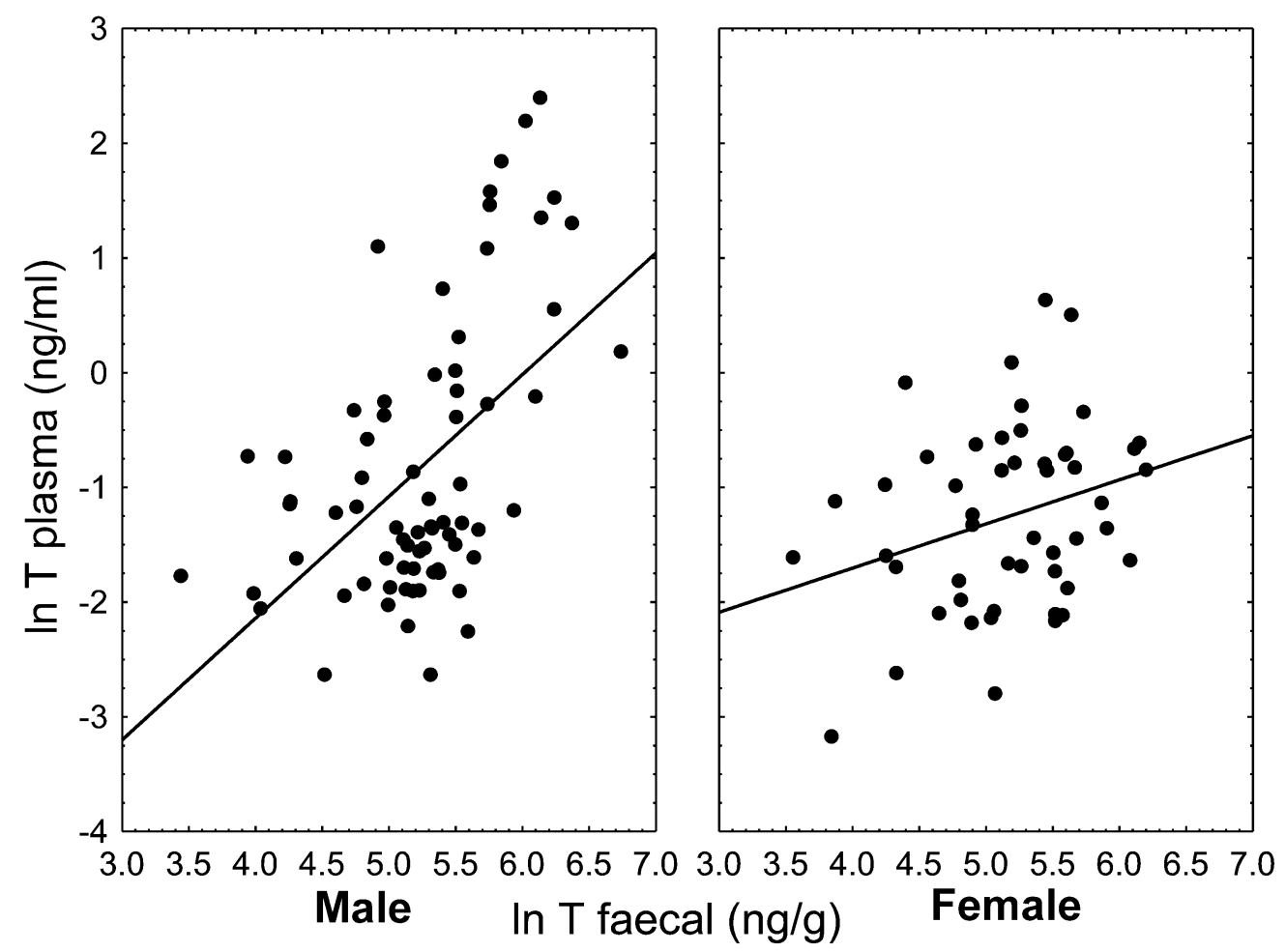

Fig 6: Correlations of $\ln$ plasma testosterone ( $\mathrm{T}$ plasma) and $\ln$ faecal testosterone ( $\mathrm{T}$ faecal) across the sexes. Male: $y=-6.385+1.061 * x ;$ Female: $y=-3.242+0.385 * x$ 